\title{
MULTICRITERIA APPROACH FOR EVALUATION OF SCENARIOS GENERATION MODELS APPLIED TO THE MEDIUM-TERM HYDROTHERMAL OPERATION PLANNING
}

\author{
Hugo Ribeiro Baldioti* \\ Electrical Engineering Department \\ Pontifical Catholic University of Rio de Janeiro \\ E-mail: hugo.baldioti@gmail.com \\ Bruno Agrelio Ribeiro \\ Electrical Engineering Department \\ Pontifical Catholic University of Rio de Janeiro \\ E-mail: agrelio@gmail.com \\ Reinaldo Castro Souza \\ Electrical Engineering Department \\ Pontifical Catholic University of Rio de Janeiro \\ E-mail: reinaldo@ele.puc-rio.br
}

\begin{abstract}
Multicriteria approach is used in the context of evaluation of synthetic scenarios generation models, aiming to rank them according to their performance by considering solely the stochastic aspects. Alternative inflow time series scenarios are generated via stochastic linear models fitted to existing historical data. These studies are motivated by the ongoing necessity of investigating improvements in the current models. The idea is the development of an index capable of sorting several alternative models that have been proposed in the literature. For that, Analytic Hierarchy Process (AHP) has been used in this paper in order to assist the adoption of the best scenarios generating model. The problem is first hierarchically structured in four levels, which is the base for the complete proposed model that includes also the four Brazilian energy subsystems and the two groups of scenarios used in the simulations and the optimization stage. The selected subcriteria are grouped into criteria which represent statistical tests chosen to assess the models that are being judged (alternatives). The weights generating process was obtained through the participation of Brazilian electrical sector specialists [National Operator of the Electrical System (ONS) amongst others], aiming at producing a consistent and reliable method for selecting the procedure that carries the specialists consensus opinion. The suggested approach produced efficiency at ordering the models and indicates, through attributes sensitivity analysis, the robustness of the method.
\end{abstract}

Keywords: multicriteria, AHP, scenarios evaluation, energy planning. 


\section{Introduction}

Hydroelectric plants are responsible for, approximately, $79.8 \%$ of the power capacity installed, while $18.8 \%$ are represented by the thermoelectric ones (the second major source). In order to coordinate, monitor and operate such complex system it is necessary to know, beforehand, the available amount of water in the reservoirs of each hydro plant in order to increase the robustness and the reliability of the electricity supply system.

Presently, in the stochastic context, each new proposal of streamflow modeling is individually evaluated. Therefore, there is no a categorical way to assess these models. In other words, once a new modeling technique is proposed it is difficult to consider whether this new approach is better or worse than the existing one. Thereby, the development of a tool capable of analyzing and sorting using selected statistical tests is highly important.

The focus of this work is the sorting, through AHP, of six synthetic scenarios generating methodologies of Natural Inflow Energy (NIE), aiming at assisting the choice of the best option to be used by the Brazilian electrical sector to model the inflow scene.

\section{Brazilian hydrothermal energetic planning context}

Having the size and the characteristics that makes it possible to be considered unique, the Brazilian production and transmission electrical system is a large scale hydrothermal one, with strong predominance of hydropower plants with multiple owners. It is an almost complete integrated grid linking the geographical subsystems: Southern, Southeastern, Midwestern, Northeastern and part of the Northern Regions.

The National Interconnected System (NIS) is split into four big subsystems: Southeast/Midwest, South, Northeast and North. Such connection is rather important to take advantage of the hydrological seasonality of the rainfall sequence, which varies considerably among them.

The electrical operation planning has as the main target the minimization of the operating costs of the system. Regarding hydroelectric and thermal power usage, the decision is taken by the National Operator of the Electrical System (for short ONS), which generates direct consequences to the final price of electrical energy and too guarantee the energy supply. In order to be able to make a decision at each stage, it is essential that the theoretical model matches the stochasticity of the physical system being modelled.

\section{Scenarios generation models}

Five among the six models that will be sorted in this paper comes from the Periodic Autoregressive $[\operatorname{PAR}(\mathrm{p})]$ family of models, which depend not only on the time lag between each observation, but also on the observed period of the year (for monthly data, there are 12 of these models, one for each month). The PAR(p) models to be considered are distinguished only in terms of noise sampling procedure. The five proposed sampling methodologies are: Log Normal; Bootstrap; Bootstrap-Monte Carlo; Bootstrap-Fuzzy; and Gama. These methods, except the first one, have been proposed in literature by 
(OLIVEIRA, 2013) and (FERREIRA, 2013). The last studied methodology is the first order Periodic Gama Autoregressive [PGAR(1)]. It is a hybrid modeling that encompasses the complementary characteristics of the additive model [PAGAR(1)] and of the multiplicative model [PMGAR(1)], implemented recently by (FERREIRA, 2013).

\subsection{Performance evaluation}

Once the simulated NIE series were generated, the next step consists of an assessment of their performance in comparison with the historic NIE data. In order to make this procedure standard it is considered not only the various comparisons with the historical series, but also in relation to the model structures themselves. To do that the following statistical tests and mathematical processes are proposed: t-test; Levene's test; Kolmogorov-Smirnov's test; Wilcoxon's test (for median evaluation); skewness analysis; negative and positive sequence test; correlation; noise t-test; arch test; and Ljung-Box's test.

\section{Methodology}

These evaluation procedures are equivalent to the criteria, however in order to simplify the modeling and the amount of required judgments for the generation of weights, the tests have been gathered into five different criteria, engendering the basic hierarchical structure of the problem. This structure has been obtained after several meetings with the decision-makers involved in the process. Thus, there are five clearly defined criteria: Basic Statistics, composed by five subcriteria (t-test, Levene's test, Wilcoxon's test, skewness analysis, and Kolmogorov-Smirnov's test); Negative Sequence Analysis, composed of four metrics (sequence sum, sequence length, sequence intensity, and maximum representation); Positive Sequence Analysis, composed of metrics similar to the previous described for the negative sequence except the maximum representation metrics; Correlation Analysis, without any subcriterion; and Model Noise Analysis. The object of the study used by this last criterion is the residual obtained by the fitted models, by considering the following subcriteria: t-test; arch test and Ljung-Box's test.

Weight judgments carried out by the selected specialists have used the hierarchy introduced above. The reliability of these judgments were checked using the consistency ratio (CR), that should produce a value less than one for coherent judgments, for that, an on-line form was developed. It is worthwhile to mention that the consistency analysis is a built-in procedure that allows on-line corrections of the evaluation by the specialist whenever the coefficient indicates this need. All together, the specialists have been consulted and their judgments have been put together by the geometric mean of the weights.

It is essential to expand the hierarchy so that a global final result is achieved, referring to all of the subsystems and the two optimization simulation phases, forward (FWD) and backward (BWD). It is important mentioning that the weights of these new elements are fixed and predefined.

\section{Results}

The table below shows the weights in reference to the optimization simulation phases (FWD and BWD), the subsystems and the attributes. It also shows the final order of the alternatives. 
Table 1

Predefined and judged weights, and final order

\begin{tabular}{|c|c|c|c|c|c|c|c|}
\hline Simulation & $w_{s f b}$ & Subsystem & $w_{\text {subs }}$ & Attribute & $\boldsymbol{w}_{a}$ & Attribute & $\boldsymbol{w}_{a}$ \\
\hline$\overline{\text { FWD }}$ & 0,5 & SE/MW & 0,610 & t-test & 0,1249 & Max. NS & 0,0407 \\
\hline \multirow[t]{7}{*}{ BWD } & \multirow[t]{7}{*}{0,5} & $\mathbf{S}$ & 0,171 & Levene & 0,1224 & Length. PS & 0,0282 \\
\hline & & NE & 0,150 & Skewness & 0,0551 & Sum PS & 0,0372 \\
\hline & & \multirow[t]{5}{*}{$\mathbf{N}$} & \multirow[t]{5}{*}{0,069} & Wilcoxon & 0,0436 & Inten. PS & 0,0442 \\
\hline & & & & K-S & 0,0799 & Correlation & 0,1469 \\
\hline & & & & Length NS & 0,0600 & t-test noise & 0,0500 \\
\hline & & & & Sum NS & 0,0433 & $\operatorname{arch}$ & 0,0275 \\
\hline & & & & Inten. NS & 0,0561 & Ljung-Box & 0,0399 \\
\hline \multicolumn{5}{|c|}{ Alternatives } & & \multicolumn{2}{|c|}{ Order } \\
\hline \multicolumn{4}{|c|}{$\begin{array}{l}\text { PAR(p) - LogNormal } \\
\end{array}$} & \multicolumn{2}{|c|}{0,2148} & \multicolumn{2}{|c|}{$1^{\circ}$} \\
\hline \multicolumn{4}{|c|}{ PAR(p) - Bootstrap } & \multicolumn{2}{|c|}{0,1929} & \multicolumn{2}{|c|}{$2^{\circ}$} \\
\hline \multicolumn{4}{|c|}{ PAR(p) - Bootstrap MC } & \multicolumn{2}{|c|}{0,1919} & \multicolumn{2}{|c|}{$3^{\circ}$} \\
\hline \multirow{2}{*}{\multicolumn{4}{|c|}{$\begin{array}{l}\text { PAR(p) - Bootstrap Fuzzy } \\
\text { PAR(n) - Gama }\end{array}$}} & \multicolumn{2}{|c|}{0,1750} & \multicolumn{2}{|c|}{$4^{\circ}$} \\
\hline & \multicolumn{3}{|c|}{ PAR(p) - Gama } & \multirow{2}{*}{\multicolumn{2}{|c|}{$\begin{array}{l}0,1295 \\
0.0960\end{array}$}} & & \\
\hline PGAR(1) & & & & & & \multicolumn{2}{|c|}{$6^{\circ}$} \\
\hline
\end{tabular}

After the final sorting, it was carried out a sensitivity analysis of the attributes with higher weights or uncertainty related to the following tests: correlation analysis; t-test; Levene's test and skewness analysis. Figure 2 shows the results of the analysis for simulation FWD of the subsystem SE/MW, as this is the most important of them, responsible for approximately $70 \%$ of the nation energy consumption.

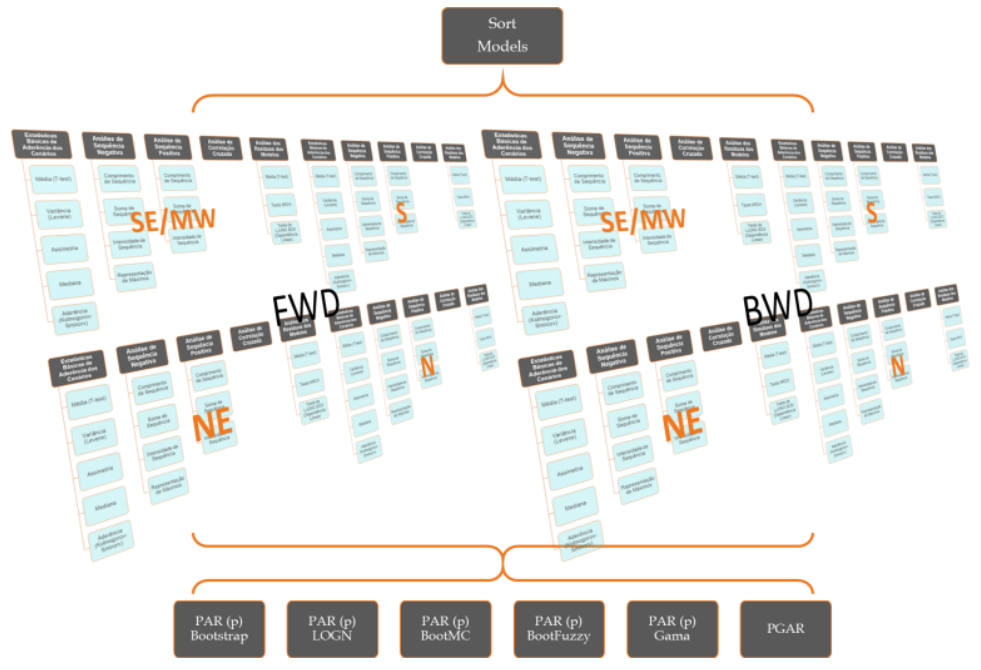

Figure 1 Complete hierarchy

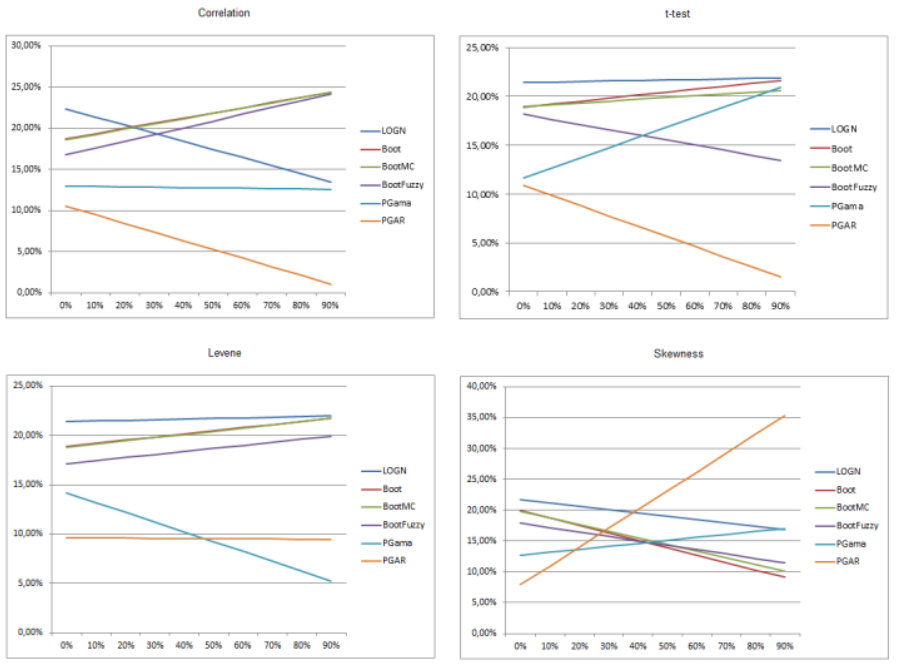

Figure 2 Sensitivity analysis

For the correlation analysis, by varying approximately $20 \%$ the value of the attribute, the PAR(p) - Bootstrap and PAR(p) - BootstrapMC models become the best choices. It is interesting to examine that the model which has presented the worst results, PGAR(1), reaches first position with an approximate variation of $40 \%$ in the skewness attribute.

International Symposium of the Analytic Hierarchy

Process
Washington, D. C. June 29 - July 2, 2014 


\section{Limitations}

It is important to mention that the consideration about the weights for the simulations creates a structural simplification, making the hierarchy linear. This means that the problem can also be treated using a different approach, such as the Analytic Network Process, once the scenarios used in the backward simulations come from the scenarios created in forward simulations, justifying the dependency structure and the non-linear hierarchy.

\section{Conclusions}

In order to meet the objectives of the comparisons, it was necessary to develop the skewness analysis and the correlation analysis, as they did not apply in the context of the traditional problem. Another implementation that was also necessary was the standardization of the assessment of the models performance. By unifying this examination it is possible to evaluate any other method for scenarios generation. The AHP presents a characteristic related to the reversal ranking, thereby it may be concluded that the ordination generated by the method is punctual and totally dependent on the considered alternatives, therefore establishing a comparative and non-global "quality index".

The fact that the final order shows the PAR(p) - LogNormal model as the best solution corroborates with the modeling proposed by the current planning of the Brazilian electrical system, however it is attained that, through sensitivity analysis, the PAR(p) Bootstrap and PAR(p) - Bootstrap Monte Carlo models are shown as good options as well.

The main contributions of this work are connected to the medium-term planning of the Brazilian electrical sector. Once the best scenarios generation model from the stochastic viewpoint is identified, the subsequent optimization process will be strongly founded based on a more robust model. It is important mentioning that the work also considers the way the scenarios are evaluated. Taking into account all that, a mechanism capable of evaluating any synthetic scenarios generation model has been created, which makes the developed tool a good option to be used by the National Operator of the Brazilian Electrical System to check on the various models proposed to generate scenarios of NIE.

\section{Key References}

FERREIRA, P. G. C., 2013. "The stochasticity associated with Brazilian Electrical Sector and a new approach to generate Natural Inflow Energy via Periodic Gama Model”. D.Sc Thesis, Pontifical Catholic University of Rio de Janeiro: Brasil.

OLIVEIRA, F. L. C., 2013. "Time Series Model for Building Scenarios Trees Applied to Stochastic Optimization". D.Sc Thesis, Pontifical Catholic University of Rio de Janeiro: Brasil.

SAATY, T. L. \& VARGAS, L. G., 2012. Models, Methods, Concepts \& Applications of the Analytic Hierarchy Process. 2nd ed. New York: Springer. 